\title{
Securing Data Quality along the Supply Chain
}

\author{
Sergej Bondar ${ }^{1}$, Christoph Ruppert ${ }^{2}$, and Josip Stjepandic ${ }^{1}$ \\ ${ }^{1}$ PROSTEP AG, Darmstadt, Germany \\ ${ }^{2}$ Heidelberger Druckmaschinen AG, Heidelberg, Germany \\ josip.stjepandic@opendesc.com
}

\begin{abstract}
Concurrent engineering in distributed development environments like those in the automotive industry places enormous demands on the organization of collaboration between the companies and departments involved. Efficient data communication in all phases of the product development process is a prerequisite for lean and flexible collaboration processes. Automotive suppliers that develop system components for a number of different OEMs or tier-1 suppliers face the challenge of ensuring that they make the CAD data available in the format required by their customers along with a high level of reliability and, if data translation is involved, that they take into consideration the system configuration of the respective customer. The main obstacle in this process chain is the insufficient data quality. This paper describes the approaches conducted in cooperation between the supplier portal www.opendesc.com and Heidelberger Druckmaschinen AG.
\end{abstract}

Keywords: CAD, Data Quality, Supply Chain, Engineering Collaboration, Migration.

\section{Introduction}

Nowadays modern manufacturing industries like the automotive are acting in the dynamic global marketplace that demands instant responses to customer requirements, short time-to-market and high flexibility in production. In the past decades the manufacturing industries were even more shifting to a distributed environment like an extended enterprise, with increasing agility. We set the customer's satisfaction on the first place in order to get the assignment against many competitors. This has caused the mass customization at high level and even more complex development, manufacturing as well as logistics processes along the manufacturing supply chain. Thus the upcoming outsourcing has derived a multi-tier supply network structure involving numerous enterprises around the globe. The product development as well takes place in global development partnerships. OEMs accomplish the development of new products at many locations in several countries across the world [1]. Furthermore, a variable number of external service providers and suppliers take part in individual projects. The most relationships in this supply network are temporary, exceed by the end of project and today's project partner can well become tomorrow's harshest competitor (Figure 1). 
The suppliers were involved in the product development as early as possible, because they mostly possess a greater depth of domain expertise for best product development. The OEM-supplier relationship is characterized by a sequential interaction, whereby the OEM gives clear product and production requirements to the supplier and the supplier delivers the product or service to the OEM. Supplier integration is a crucial method for incorporating a supplier's innovativeness in the product development process and reduces the costs and risk [2]. It also creates synergy through mutually interacting deliverables and decisions between OEM and suppliers. Both sides take advantage of each other's capability to develop the product as well as to obtain feedback from the other party to improve the product development. Due to the complex development cycle, the OEM took the lead and has begun to adopt supplier integration into its product development process. To respond to this trend, the collaboration and partnership management between the OEM and suppliers need to be continuously improved to reduce costs and time. Regarding the depth of collaboration, the integration of suppliers into the OEM process chain can be defined in many ways, depending on corresponding work package and type of collaboration [3]. To enable the success of supplier integration, this work describes how to improve the collaboration between the OEM and its suppliers, through ensuring the appropriate data quality.

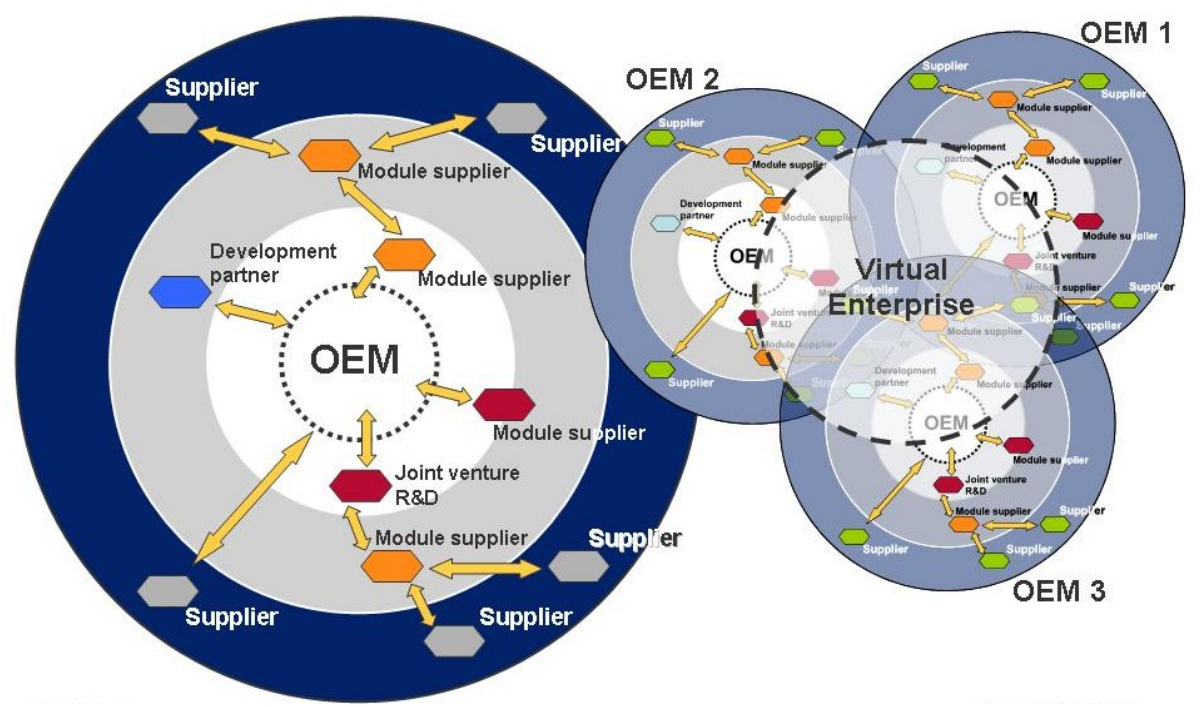

Fig. 1. Virtual enterprise in automotive industry

In the context of concurrent and collaborative engineering, the validity and consistency of product information become important. However, it is difficult for the current computer-aided systems to check the information validity and consistency because the engineers' intent is not fully represented in a consistent product model. Due to the different approaches and IT systems in the automotive OEM industry, a unified solution is not possible at this time. In particular, automotive suppliers that 
develop system components for a number of different OEMs or tier-1 suppliers, face the challenge of ensuring that they make the CAD data available in the format required by their customers and with a high level of reliability and, if data translation is involved, that they take the system configuration of the respective customer into consideration. Special requirements like the flexibility for further changes in business relationships and the intellectual property protection are also taken into account.

\section{Cooperation Models}

As the outsourcing became standard business approach in the manufacturing industries, many industry associations like the German automotive manufacturers association (VDA) accomplished the basic development work to define and classify the typical collaboration models and corresponding processes [4] (Figure 2). There are 6 supplier types defined according to the criteria: production technical integration, process integration, functional integration, and geometrical (spatial) integration of the whole product (car). Beside of the prime contractor who can be defined as a clone of the OEM without the product management, sales and marketing function, all other types of suppliers (system supplier, module supplier, component supplier, part supplier, and engineering service provider) maintain a high level of independency in their corresponding processes. Taking the fact into account that a supplier supplies many customers, who have their own, various processes and infrastructures, this creates a strong need for a comprehensive integration approach based primarily on standards and serving the relationships to all the customers.

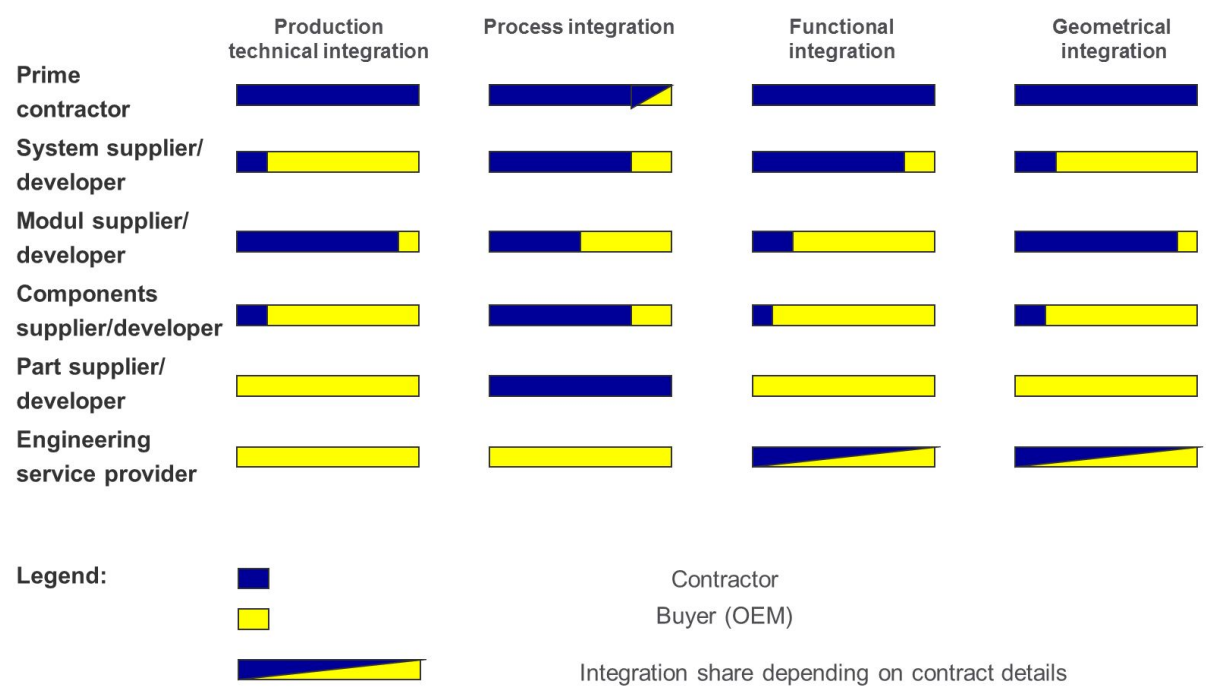

Fig. 2. Cooperation models in the automotive industry

The responsibility of suppliers to provide the suitable product documentation in each phase of product emerging process is self-evident for an OEM. Thus the supplier 
is forced to take the corresponding measures to adapt his data to OEM's requirements. The most important criterion is the CAD data quality as defined and required by OEM.

\section{CAD Data Quality}

In the product, various life-cycle quality requirements at the particular stages are defined and required. These include requirements such as the functional requirements of the product, the type of presentation form, the production requirements or the data quality in the data exchange.

Within the design and manufacturing process chains, there are different phases that shape up demands on the geometric and organizational data quality and the data size. A CAD model can both find multiple uses as well as require certain compatibility. The multiple use is mainly based on the modeling, while the compatibility refers more to the geometrical and topological characteristics. In downstream or subsequent applications, it may happen that the designer says, the CAD model would be good, while the downstream users of this CAD model in the process chain often described it as not so good or even unusable. The same situation can occur in the collaboration context.

Therefore $\mathrm{CAD}$ data should ensure the reusability in native CAD system, but also the derivation of the resulting geometry for downstream data processing. Although the level of detail can be low or high during a certain period, the requirements remain the same for geometry and topology.

A categorization of CAD data yields the matching of criteria and characteristics to reasonable and memorable sets of 3D CAD models. There are different approaches, such as the division into "components" or "resources" (for producing components), in various process chains (depending on the material or the manufacturing method) or in accordance with the stages of development (concept, coordination, approval, detailing, etc.). The working group of German Automotive Industry Association (VDA) recommends a two-dimensional matrix [5]. Meanwhile, these basic criteria are adopted by almost all OEMs.

Finally the term "CAD data quality" includes the fulfillment of a set of predefined criteria known as mathematical-technical and organizational. To the first set belongs the geometrical quality of CAD models and depends on fulfillment of basic accuracy criteria (coincidence, continuity), itself depending on accuracy setup of CAD system. The second set comprises the relevant rules for the structure of CAD models. Almost each large company has defined own CAD methods which include a set of prerequisites. The fulfillment of the rules is checked by special CAD checkers, which are controlled by profiles for each model purpose.

For the translation from the original into the target $\mathrm{CAD}$ format, direct or neutral interfaces are used. The advantage of direct interface is the higher translation speed. The neutral interfaces are more robust by using special healing algorithms for corrupt geometry and allow better logging by using neutral format like STEP and JT.

The main problem in the CAD translation is in the most cases the insufficient data quality after the translation. The requirement for the successful translation process can also be redefined as fulfillment of all relevant data quality requirements, independent of the source system and the stage of the product development process. In the context of collaboration, this requirement can be extended to a level at which the CAD 
conversion must successfully pass the examination with each $\mathrm{CAD}$ checker on the target site, independent from the source CAD system [6].

\section{Solution Approach}

Since the problem has been identified and analyzed, now a concept for ensuring data quality in CAD data exchange has to be developed. For this, only measures are recorded to ensure data quality, and then, finally, to apply the phase model for development of methods.

Measures to prevent loss of information can be taken by considering functional aspects, verification aspects and application aspects. The functional aspects include the division of the total amount of an element specification in compatible element subsets that are adopted in individual performance levels with their associated functional content. The verification of aspects includes procedures to verify and validate the performance of a specification.

The application aspects include specific requirements such as the implementation of a new interface. Due to the wealth and diversity of the internal data models of CAD systems a loss of information during the data exchange cannot be completely avoided. Therefore, specific agreements between data sender and receiver must contribute to minimize the loss of information in the data exchange. Appropriate checklists are required and the criteria can be derived for boundary conditions. These checklists should be created as a function of the gradual approach of the functional performance of the interface [7].

The methodological concept development can be understood as decision making process and consists of a sequence of iterative steps. These steps include extensive analytical and synthesizing activities and the appropriate documentation of the analysis and synthesis results. A methodical approach is necessary because all possible requirements have to be considered in a process. Analogous to the phases for the implementation of IT projects, the concept phase, the specification phase, and the implementation phase of development are to be introduced as part of the methodological concept development to ensure data quality,

The essential task in the concept phase is the creation of requirements profile, which includes the performance range. The requirements profile includes a checklist, or a sort of guide for meaningful modeling technique that could prevent problems in data exchange. Herein the pre-mentioned causes are considered. This checklist has been created after many investigations and has been continuously adapted to users.

Based on extensive experience, the necessary guidelines were defined similarly to the VDA recommendation 4955 [5]. Here best practices are set up or attempting to establish common guidelines that are either independent of the used CAD systems or specifically dependent on system pairing (e.g. CATIA (Creo) $\rightarrow$ NX), processors and interface formats (e.g. STEP).

The implementation of design guidelines must be established by appropriate check tools. The application is done by native checking capabilities, following the progress of the design process, pointing mainly at the possible errors in data exchange, what is therefore very helpful. The final check can be done in the batch mode. The positive result is the prerequisite for the release of data exchange. 
The check tools are modular. The runs by the user are executed individually or in any combination. Limits and other underlying assets for each run will be proposed through a configuration file and can be changed interactively. A selection or limitation of scope of testing is possible, but just a test of all the elements finally matters.

The logging of the test run, including indication of corrupt elements is in a log file. The respective defective items must be clearly identifiable.

Further, the display of certain entities (e.g. transferred and arrived surfaces) from the log file need to be written out after the data export to instantly identify potential losses (e.g. surface count). This required comparative studies to be made with respect to the different $\log$ files of the pertaining CAD systems and to the meaningfulness of the $\log$ files.

Finally, a figure is required, indicating the number of transferred data elements during the export of native data formats to the neutral file and potential losses as well. This is a comparison between the elements between the states "before" and "after".

The final concept is shown in Figure 3.

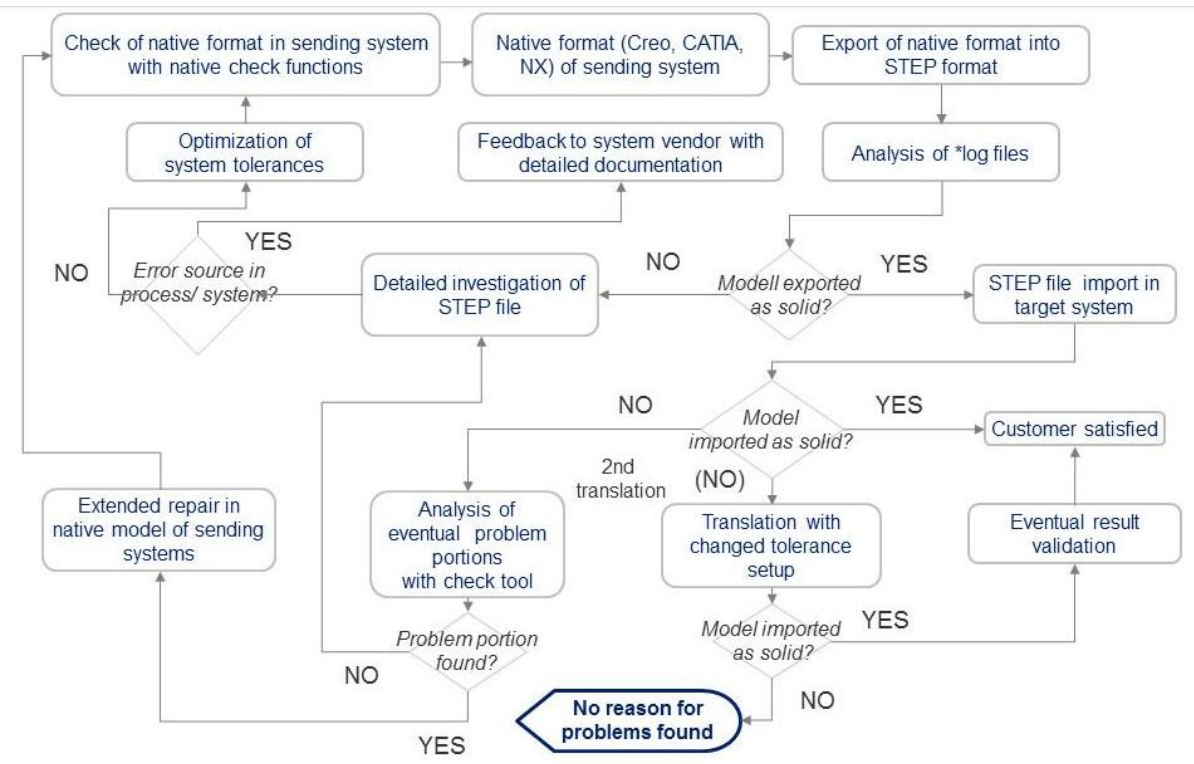

Fig. 3. Final concept for the test procedure

The validation phase is used to check and, if necessary, to improve the concept specification. It must be checked whether the developed method meets the requirements profile from the concept phase. To perform the test, it is necessary to establish criteria and procedures. Based on test criteria and methods, check tools can be developed that allow computer-aided validation. The result of the validation phase can eventually lead to the revision of the concept. 


\section{$5 \quad$ Validation}

Validation methods target the proof of the validity of the specification and implementation. They use the methods for verification, confirming the completeness and correctness of a system with respect to a reference system, and the methods of falsification, which serve to demonstrate the faultiness of a system. For validation, test methods are used, which are employed to demonstrate conformity. For the detection of a stable behavior, one applies check methods, which are used for qualitative examination of behavior at different levels of complexity in accordance with predetermined criteria. Finally, measurement methods are used for the quantitative determination concerning the behavior of the basis of predetermined measurement criteria.

The use of validation methods is different in the different phases of the product development model. Depending on the degree of product specification model description, based on the reference model, the formal specification and the implementation, validation procedures can be used in an increasingly quantifying way. In our case, all methods have been incorporated into a fully automated workflow, which is based on the software OpenDXM from PROSTEP (Figure 4). Thus the highest level traceability is achieved [8].

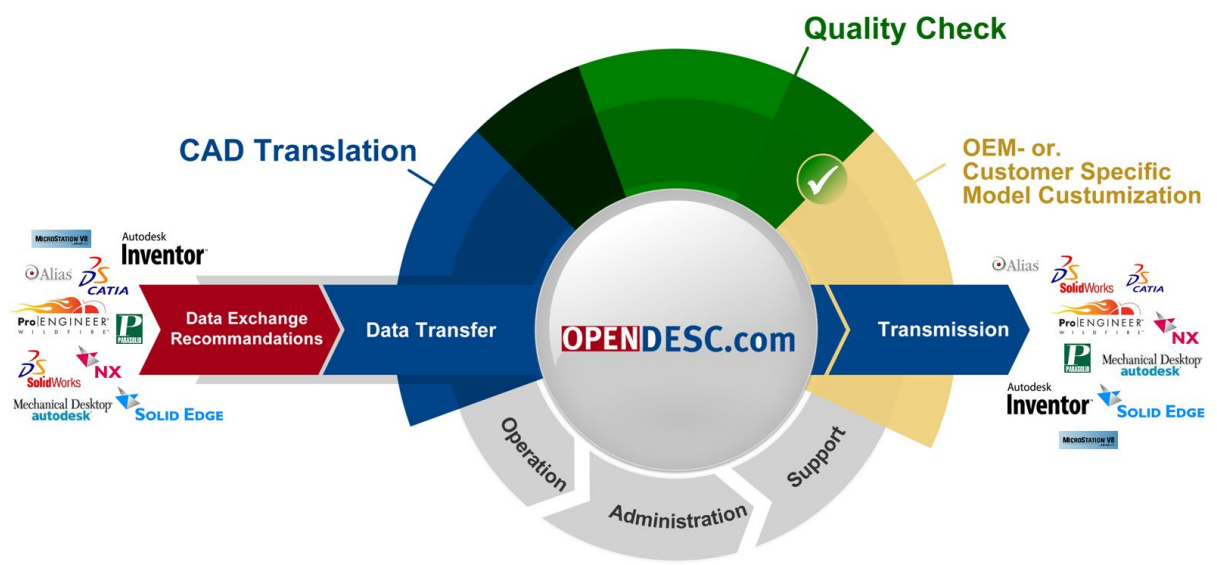

Fig. 4. CAD translation in www.opendesc.com

\section{$6 \quad$ Use Case Daimler}

Since Daimler has taken the decision to switch to CAD system NX of Siemens PLM instead of CATIA V5 of Dassault Systemes there is a huge change in the customer process of almost each supplier. The overall situation is shown in Figure 5. Each supplier has to serve simultaneously two target systems (CATIA for ongoing and NX for upcoming projects) with the same or similar content [9]. This procedure includes many CAD translation steps (scenarios 4 to 9) which are in principal not beneficial for good data quality. The challenge is in preserving such a level of data quality to ensure that all translating processes are successful. The data quality in CATIA is 
checked with QChecker again, in NX by using the newly deployed Heidelberg CAx Quality Manager (HQM).

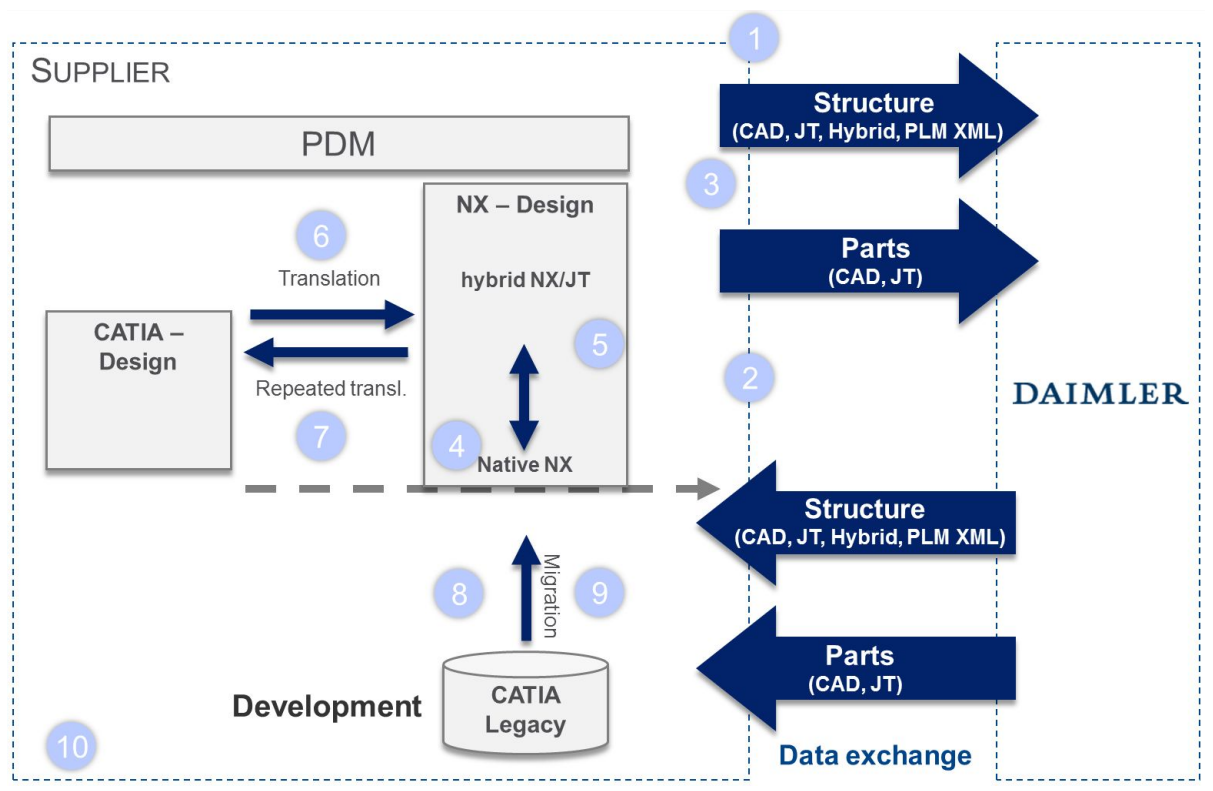

Fig. 5. The change in collaboration between Daimler and suppliers

\section{$7 \quad$ Results}

The procedure shown above was applied for different use cases, including different design content (powertrain, interior, electrics, chassis). Most of them were Creo data, modeled and prepared for translation to CATIA V5, as actually used in ongoing development projects at Daimler. Therefore, a good comparability between "old scenario" and "new scenario" is given.

The translators in CATIA and NX disclose the similar level of performance and robustness. The base system tolerance lies with $0,001 \mathrm{~mm}$ at the same level. Initially, all models can be transferred lossless without exception to CATIA and NX. However, it appears in some cases that automatic healing algorithms have slightly adjusted the geometry to satisfy the continuity condition. To what extent this would lead to further problems in further processing, could not be definitely predicted.

Further comparisons in the model properties like center of gravity, moments of inertia, as well as cloud of points were also within the allowed tolerances and showed no abnormalities.

Further investigations were accomplished with the models that once have already been converted, because that is the typical implication of complex scenario shown in Figure 5. Here occurred considerable problems and losses that had to be corrected manually. Such models generally reveal significant quality problems and should be avoided. The Heidelberg CAx Quality Manager (HQM) was very helpful to identify the problems and make a repair easier; a typical application is shown in Figure 6. 


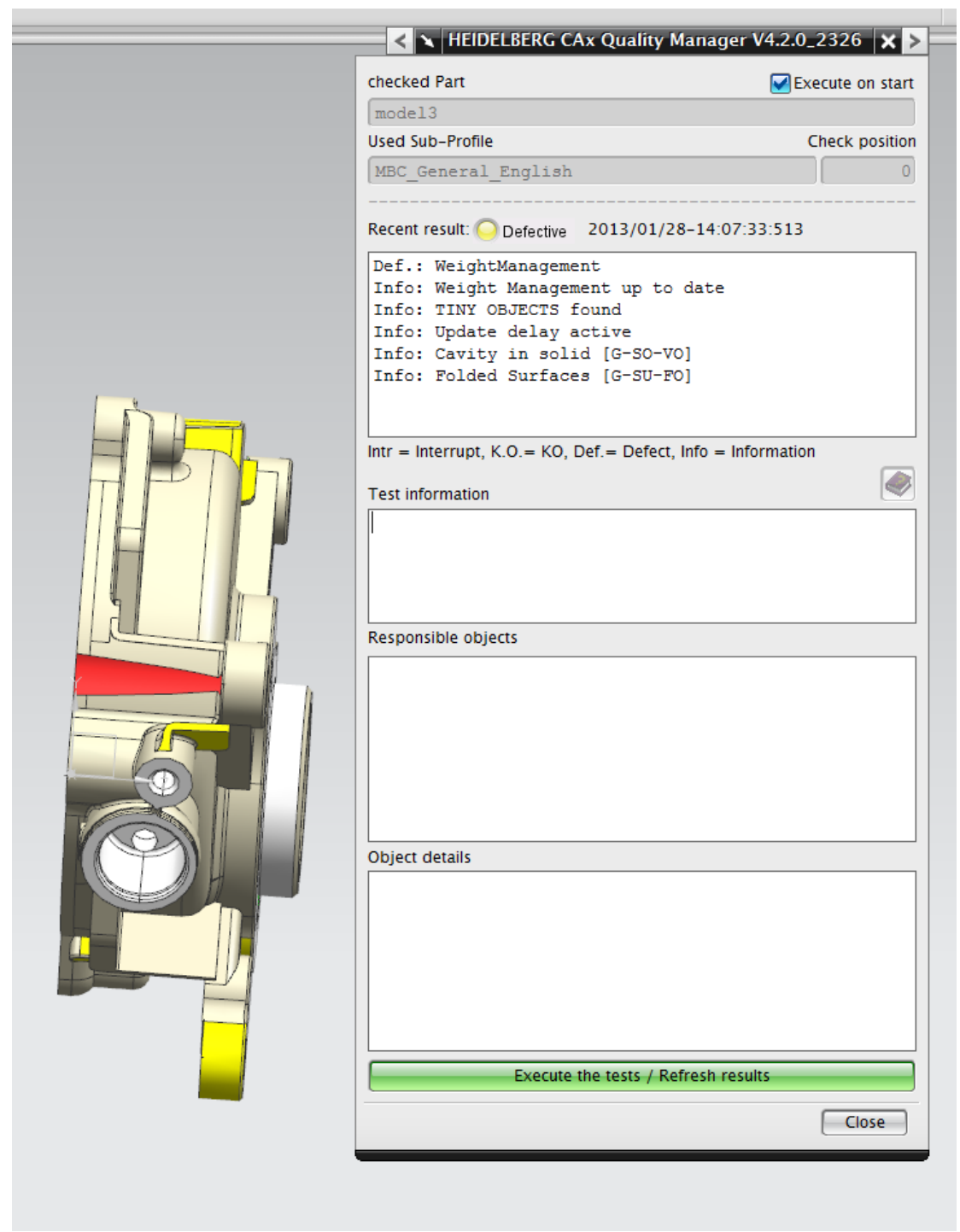

Fig. 6. Typical results in Heidelberg CAx Quality Manager

\section{Conclusions and Outlook}

In a dynamic collaborative environment like global automotive industry the working conditions are subject to a continuous change. Suppliers who work together with different OEMs and tier-1 suppliers have to constantly cope with new requirements relating to exchange partners, data formats, system environments to be supported, quality and security requirements, etc. If they take data communication with customers into their own hands, this means that they have to constantly adapt their 
data translation and exchange processes to the ever-changing requirements. This involves considerable administrative overhead in terms of time and money, which can occasionally have a negative impact on quality and adherence to deadlines. Collaboration with a competent service provider is therefore an interesting alternative, as it not only cuts costs, but also facilitates making the exchange processes uniform and ensures a higher level of reliability and traceability.

Good example for the long-term stability is the proposed approach to support the recent move of Daimler by replacing CATIA with NX. While many suppliers, which provide the data communication on their own, are forced to adapt their infrastructure, processes and methods to new environment at Daimler by spending a significant amount of money and time, the proposed solution allows to work again in the same environment by using predefined interface in the customer process like supplier portal www.opendesc.com. The check tool Heidelberg CAx Quality Manager (HQM) performs a significant contribution to successful data exchange.

The weakness of the proposed solution is the lack of solution for parametric data translation which still has to be developed.

The future development belongs the further automation of whole communication process and provision of "communication plugin" [10] for each OEM, based on recent standards (STEP AP242 and JT), in order to avoid the expensive point-to-point connection.

\section{References}

1. Stark, J.: Global Product. Strategy, Product Lifecycle Management and the Billion Customer Question. Springer, London (2007)

2. Dyer, J.H.: Collaborative advantage: winning through extended enterprise supplier networks. Oxford University Press, Oxford (2000)

3. Tang, D., Chin, K.-S.: Collaborative Supplier Integration for Product Design and Development. In: Wang, L., Nee, A.Y.C. (eds.) Collaborative Design and Planning for Digital Manufacturing, pp. 99-116. Springer, London (2009)

4. N.N: VDA Empfehlung 4961/3, Abstimmung der Datenlogistik in SE-Projekten, VDA, Frankfurt (2012)

5. N.N: VDA-Empfehlung 4955 Umfang und Qualität von CAD/CAM-Daten, Version 3, VDA, Frankfurt (2002)

6. Bondar, S., Potjewijd, L., Stjepandic, J.: Globalized OEM and Tier-1 Processes at SKF. In: Stjepandic, J., et al. (eds.) Concurrent Engineering Approaches for Sustainable Product Development in a Multi-Disciplinary Environment, pp. 789-800. Springer, London (2013)

7. Fischer, T., Martin, H.P., Endres, M., Stjepandic, J., Trinkhaus, O.: Anwendungsorientierte Optimierung des neutralen CAD-Datenaustausches mit Schwerpunkt Genauigkeit und Toleranz, VDA, Frankfurt (2000)

8. Reim, R., Cordon, P., Hund, A., Stjepandic, J.: CAD-Konvertierung: Motivation, Probleme, Lösungen, CADCAM Report, 9 (2007)

9. Wendenburg, M.: Konvertierung als Dienstleistung, CADCAM 2007, 9-10 (2007)

10. Mervyn, F., Kumar, A.S., Nee, A.Y.C.: A 'Plug-and-Play' Computing Environment for an Extended Enterprise. In: Li, W.D., et al. (eds.) Collaborative Product Design and Manufacturing Methodologies and Applications, pp. 71-91. Springer, London (2007) 